\title{
PREDICCIÓN DE LA ESTRUCTURA DIAMÉTRICA DE ESPECIES COMERCIALES DE UN BOSQUE SUBTROPICAL POR MEDIO DE MATRICES DE TRANSICIÓN
}

\author{
Susana Mariela Teresczcuch*, Patricio Miguel Mac Donagh**, Adrián Javier de Oliveira***, \\ Liliana Elizabeth Rivero*, Nardia Maria Luján Bulfe* \\ *Ing. Forestal. Facultad de Ciencias Forestales, UNaM, Misiones, Argentina - mteresczcuch@facfor.unam.edu.ar - \\ li_rivero@yahoo.com.ar-nardia@facfor.unam.edu.ar \\ **Ing. Forestal, M. Sc. Facultad de Ciencias Forestales, UNaM, Misiones, Argentina - mdonagh@facfor.unam.edu.ar \\ ***Ing. Forestal, Mazter Industrial Maderil S.A. - deoliveiraadrian@yahoo.com.ar \\ Recebido para publicação: 20/09/2005 - Aceito para publicação: 13/12/2006
}

\begin{abstract}
Resumen
El presente trabajo tuvo por objetivo predecir la distribución diamétrica a través de matrices de transición para un bosque nativo sometido a dos metodologías de cosecha: convencional e impacto reducido. Se comparó la incidencia de los tratamientos respecto a la evolución del área basal inicial y número de individuos. Se utilizaron datos provenientes de inventarios de parcelas permanentes medidos en el período 1998 - 2001 y 2004. Como resultado se verificó que las predicciones realizadas por las matrices de transición no difieren del número real de árboles por clase diamétrica a un nivel de significancia de 0,05 . A través de las simulaciones queda demostrado que el tratamiento de cosecha de impacto reducido recupera su área basal original a los 21 años de realizado el aprovechamiento y el número de árboles/ha a los 12 años; el tratamiento de cosecha convencional recupera el área basal original a los 24 años y el número de árboles/ha a los 21 años. Esta diferencia se debe a que los criterios fijados para el tratamiento de cosecha de impacto reducido produjeron una disminución en los daños a la masa remanente principalmente a las especies de interés comercial, asegurando la permanencia para futuras cosechas.
\end{abstract}

Palabras claves: Manejo forestal; tratamientos de cosecha; impacto reducido; área basal; Selva Paranaense.

\begin{abstract}
Summary
Prediction of the diameter structure of commercial species in a subtropical forest through transition matrix. This work had the objective of predicting the diameter distribution of a neotropical forest subjected to two levels of harvesting through the transition matrix techniques. The harvesting treatments were conventional logging and reduced impact logging. It were compared the intensity of the harvesting treatments in relation to the evolution of the initial basal area and number of trees. The data used came from of permanent plots measured in the periods 1998 - 2001 and 2004. It was verified that the predictions by the transition matrix do not differ from the real number of trees by diameter class at 0.05 significance level. The simulations demonstrated that the reduced impact logging recovers its original basal area and number of trees in 21 years and 12 years, respectively; the conventional harvesting recovers the original basal area in 24 years and the tree numbers in 21 years. This difference between both treatments are explained because the reduced impact treatment produced a decrease in the damages to remainder trees, mainly to the commercial species, ensuring through this the trees for future harvests.

Keywords: Forest management; harvesting treatments; reduced impact logging; basal area; Atlantic Forest.
\end{abstract}

\section{INTRODUCCIÓN}

El aprovechamiento forestal es considerado muchas veces como el primer tratamiento silvicultural destinado al manejo de un bosque (LOUMAN, 2001). En este sentido la base para realizar un manejo sostenible es una correcta planificación de las operaciones de cosecha. En el aspecto forestal esta planificación debe contemplar la predicción de rendimientos en madera y los turnos de corta. 
En las últimas décadas se han desarrollado conceptos y métodos de cosecha de impacto reducido (DYKSTRA; HEINRICH, 1996). Las técnicas y pautas de cosecha de impacto reducido no son reglas fijas pero se desarrollan de acuerdo con objetivos de administración, con las características del ecosistema y las condiciones del mercado. Los sistemas de cosecha de impacto reducido se adaptan a técnicas basadas en actividades como ser un inventario de pre-cosecha, la ubicación de árboles seguido de una planificación de caminos y sus vías de saca y una tala direccional a toco bajo.

Existen varias técnicas para cubrir los aspectos referidos a la predicción del rendimiento, entre las que se pueden mencionar las matrices de transición (SANQUETTA et al., 1996; AZEVEDO et al., 1994; CUNHA et al., 2002).

Según Scolforo (1997), entre los modelos por clase de diámetro la matriz de transición es un importante instrumento para viabilizar una prognosis de la producción en un bosque nativo.

La matriz de transición podría ser utilizada para simular las consecuencias de intervenciones programadas por el ordenador forestal, auxiliándolo en la toma de decisiones tratando de optimizar la utilización de los recursos forestales. Este modelo, si es aplicado de manera eficiente, podrá constituir una herramienta de gran valor para la búsqueda de soluciones relativas al manejo en el régimen del rendimiento sostenido de los bosques naturales (SANQUETTA et al., 1995). Puede ser utilizado para elaborar simulaciones del desarrollo futuro del bosque después de una explotación y estudiar el efecto de diferentes tratamientos y opciones de manejo sobre las tasas de crecimiento de la población (AZEVEDO et al., 1994).

A partir de las proyecciones de las frecuencias diamétricas del bosque, se podrán hacer proyecciones referentes al manejo de la producción del bosque natural tales como predicciones de volumen y área basal, entre otras. La proyección de la estructura diamétrica en el tiempo es de real importancia para el manejo y la economía de los bosques nativos (SANQUETTA et al., 1995).

Sanquetta et al. (1996) demostraron que para un bosque natural cortando los árboles mayores a $30 \mathrm{~cm}$ de D.A.P. en 1984, serían necesarios 12 años para que el bosque recupere su frecuencia inicial.

Pulz et al. (1999) concluyeron que la prognosis de la estructura diamétrica utilizando la matriz de transición se mostró eficiente a partir de tres diferentes períodos de medición del bosque, a pesar de haberse detectado cambios en la estructura del bosque, particularmente en la tasa de reclutamiento y en la mortalidad.

El objetivo de este trabajo fue predecir la distribución diamétrica a través de matrices de transición para un bosque nativo sometido a dos metodologías de cosecha. Comparar la incidencia de los tratamientos de cosecha respecto a la evolución del área basal inicial y del número de individuos por hectárea.

\section{MATERIAL Y MÉTODOS}

\section{Caracterización del bosque}

El presente trabajo fue desarrollado en la Reserva de Uso Múltiple Guaraní ubicada dentro de la Reserva de Biosfera Yabotí, en la provincia de Misiones, Argentina. Su posición geográfica es de $26^{\circ} 56^{\prime}$ Sur y $54^{\circ} 15^{\prime}$ Oeste.

El área de estudio presenta vegetación del bosque subtropical original conteniendo una gran diversidad vegetal, árboles, arbustos, bambúceas, helechos, plantas trepadoras y epifitas. Garibaldi et al. (2000) encontraron 102 especies arbóreas 79 géneros y 42 familias botánicas, destacándose principalmente Leguminosas (14\%), Lauráceas (13\%), Rutáceas $(9 \%)$ y Meliáceas (8\%). La densidad promedio es de 277 árboles y 46 especies por hectárea.

El clima de la región corresponde al tipo Cfa, según la clasificación de Köeppen, que es mesotérmico, constantemente húmedo y subtropical, con precipitaciones anuales de 1700 a $2400 \mathrm{~mm}$, distribuidas en todos los meses del año. La temperatura media del mes más cálido (enero) es de $25^{\circ} \mathrm{C}$ con máximas de $39^{\circ} \mathrm{C}$, y la temperatura media del mes más frío (julio) es de $14{ }^{\circ} \mathrm{C}$, con mínimas absolutas de $-6{ }^{\circ} \mathrm{C}$.

Se instalaron en el área bajo estudio 18 parcelas de $200 \times 200$ metros cada una, las cuales contaban con una parcela central de 100 x 100 metros, donde se relevaron todos los ejemplares arbóreos y palmas mayores a $10 \mathrm{~cm}$. de diámetro a la altura del pecho (D.A.P.) antes de la explotación (1998) y luego de las remediciones post-aprovechamiento, las cuales se realizaron anualmente durante el período 
2000-2004. La superficie total del ensayo fue de 72 hectáreas de las cuales 60 tuvieron modalidades de aprovechamiento diferentes: tratamiento de cosecha convencional, tratamiento de cosecha de impacto reducido, contando también con parcelas testigo (MAC DONAGH et al., 1999) (Tabla 1).

Tabla 1. Descripción de los tratamientos aplicados.

Table 1. Treatments summary.

\begin{tabular}{lccc}
\hline Tratamientos & Cosecha convencional & Cosecha de impacto reducido & Testigo \\
\hline Descripción & $\begin{array}{c}\text { El obrajero debió } \\
\text { seleccionar los } \\
\text { individuos a cortar, } \\
\text { estableció los recorridos } \\
\text { y las planchadas dentro } \\
\text { de cada parcela. }\end{array}$ & $\begin{array}{c}\text { Fundamento: aplicar criterios de } \\
\text { sustentabilidad en la selección de los } \\
\text { árboles a cortar. En función de esto se } \\
\text { estableció el recorrido de la } \\
\text { maquinaria dentro de la parcela, la } \\
\text { ubicación de las vías de saca y las } \\
\text { planchadas. Se establecieron las } \\
\text { intensidades de tránsito permisibles } \\
\text { para cada sector del terreno. }\end{array}$ & $\begin{array}{c}\text { árboles y no se } \\
\text { realizó ningún } \\
\text { tránsito con } \\
\text { maquinaria }\end{array}$ \\
\hline Número de parcelas & 6 & 9 & 3 \\
\hline AB extraída $\left(\mathrm{m}^{2} / \mathrm{ha}\right)$ & 1,90 & 1,36 & - \\
\hline
\end{tabular}

Para la elaboración del presente trabajo fueron consideradas únicamente las especies comerciales.

En la tabla 2 se presentan las distribuciones diamétricas iniciales (1998) antes de la explotación para cada tratamiento de cosecha y para las parcelas sin intervención.

Tabla 2. Frecuencias diamétricas iniciales de los tratamientos.

Table 2. Treatments initial frequency diameter.

\begin{tabular}{|c|c|c|c|}
\hline \multirow{2}{*}{$\begin{array}{l}\text { Centro de clase } \\
\text { diamétrica }(\mathrm{cm})\end{array}$} & \multicolumn{3}{|c|}{$\begin{array}{c}\text { Frecuencia inicial } \\
\text { (Individuos/ha) }\end{array}$} \\
\hline & Cosecha convencional & Cosecha de impacto reducido & Testigo \\
\hline 15 & 22,3 & 21,6 & 28,3 \\
\hline 25 & 23,0 & 22,6 & 26 \\
\hline 35 & 22,2 & 19,0 & 26 \\
\hline 45 & 14,6 & 15,8 & 17 \\
\hline 55 & 9,2 & 7,4 & 7 \\
\hline 65 & 3,9 & 4,9 & 6 \\
\hline$\geq 70$ & 5,6 & 3,8 & 6 \\
\hline Total & 100,8 & 95,1 & 116,3 \\
\hline
\end{tabular}

Para establecer si el bosque era homogéneo previo a la cosecha, se analizaron las diferencias en el número de individuos entre las parcelas en las cuales se aplicaron los tratamientos de cosecha y las testigos. Para ello se realizó un análisis de varianza por medio del test no paramétrico Kruskal-Wallis, a un nivel de significancia de 0,05 (Morales, 2005). El test mostró que en los tratamientos de cosecha y en las parcelas testigo no existen diferencias en el número de árboles al $95 \%$ de confianza (p-valor $>0,05$ ). Donde: $\chi^{2}$ teórico $=5.99 ; \chi^{2}$ calculado $=1.03 ; \mathrm{gl}=2$.

\section{Análisis de datos}

Matriz de transición original

Las Matrices de Transición para las parcelas testigos fueron construidas de la siguiente manera:

$$
(\mathrm{A}) \cdot\left(\mathrm{E}_{0}\right)+(\mathrm{R})=\left(\mathrm{E}_{1}\right)
$$


Donde:

(A) = Matriz que contiene las probabilidades de transición.

$\left(\mathrm{E}_{0}\right)=$ Vector de stock en el momento cero, es decir, la distribución diamétrica antes de la predicción.

$(\mathrm{R})=$ Vector de reclutamiento (ingresos).

$\left(E_{1}\right)=$ Vector de stock en el momento uno, en el tiempo de predicción futuro.

Obtención de las probabilidades de transición:

$$
\begin{aligned}
& \mathrm{a}_{\mathrm{j}}=\mathrm{N}_{\mathrm{j} 0} / \mathrm{A}_{\mathrm{j}} \rightarrow \text { (Coeficientes de la diagonal inferior) } \\
& \mathrm{m}_{\mathrm{j}}=\mathrm{M}_{\mathrm{j} 0} / \mathrm{E}_{0} \\
& \mathrm{a}_{\mathrm{j}}=1-\mathrm{b}_{\mathrm{i}}-\mathrm{m}_{\mathrm{j}} \rightarrow \text { (Coeficientes de la diagonal principal) }
\end{aligned}
$$

Donde:

$\mathrm{a}_{\mathrm{j}}$ : Probabilidad de transición a la clase siguiente inmediata.

$\mathrm{m}_{\mathrm{j}}$ : Probabilidad de muerte durante el tiempo de permanencia en la clase.

$a_{\mathrm{j}}$ : Probabilidad de permanencia en la clase.

$\mathrm{N}_{\mathrm{j} 0}$ : número de árboles que transitan en el estado ${ }_{i}$, obtenido entre medición (año 0 ) y remedición (año 1) en cm.

$\mathrm{A}_{\mathrm{j}}$ : Número de árboles existentes en la clase diamétrica ${ }_{\mathrm{i}}$ en el tiempo considerado.

$\mathrm{M}_{\mathrm{j} 0}$ : Árboles muertos naturalmente dentro del período comprendido entre $0 \mathrm{y}$ la remedición, para $\mathrm{J}$.

j: Clases diamétricas estudiadas.

: Momento inicial del período de crecimiento considerado.

\section{Matriz de transición después del corte}

Sanquetta et al. (1996), proponen un modelo de simulación para una masa de bosque nativo sometida a una explotación, en el cual se consideran los siguientes supuestos:

- Después de la cosecha ocurre la mortalidad por daño, ya que al ser apeados los árboles de gran porte, éstos alcanzan árboles en sus periferias. Esto ocurre en forma inversamente proporcional al D.A.P.

- La tasa de mortalidad natural se mantendrá igual a la tasa verificada antes de la explotación.

- La apertura del dosel creada al retirar los árboles de mayor diámetro provocará una aceleración sobre el crecimiento diamétrico de los remanentes, lo cual también es inversamente proporcional al D.A.P.

- Con el paso del tiempo, la tasa de aceleración del crecimiento irá disminuyendo en forma proporcional a la tasa de recuperación del área basal, llegando a cero en el momento en que el bosque recupere su área basal original.

- La apertura del dosel provocará también un aumento en el reclutamiento a causa del aumento de la luminosidad en el bosque.

Las matrices de transición para los tratamientos de cosecha convencional y cosecha de impacto reducido fueron construidas utilizando esta metodología.

Si un bosque sufre intervenciones se esperan cambios en los componentes de la matriz de transición (A). El modelo aquí propuesto tiene por objetivo agregar al modelo original cambios en función de la intervención programada. Más específicamente los siguientes componentes serán alterados:

- Vector de stock (E’): deberá reflejar el número de árboles (u otra variable de interés por ejemplo área basal) después de realizado el corte y la mortalidad por daño.

- Vector del reclutamiento $\left(\mathrm{R}^{\prime}\right)$ : deberá reflejar la aceleración en la tasa de reclutamiento debido a la apertura del dosel.

- Matriz de transición (A'): deberá reflejar la aceleración en el crecimiento a consecuencia del aumento en la tasa de iluminación producido por la apertura del dosel.

A continuación se presenta el modelo matricial, representado de la misma forma que el modelo original, pero con sus componentes alterados según la metodología descripta en los siguientes ítems: 


$$
\left[\begin{array}{cccc}
a_{11}^{\prime} & a_{12}^{\prime} & \cdots & a_{1 j}^{\prime} \\
a_{21}^{\prime} & a_{22}^{\prime} & \cdots & a_{2 j}^{\prime} \\
\vdots & \vdots & \cdots & \vdots \\
a^{\prime} & a_{i 2}^{\prime} & \cdots & a_{i j}^{\prime}
\end{array}\right] \cdot\left[\begin{array}{l}
e_{1}^{\prime} \\
e_{2}^{\prime} \\
\vdots \\
e_{i}^{\prime}
\end{array}\right]+\left[\begin{array}{l}
r_{1}^{\prime} \\
r_{2}^{\prime} \\
\vdots \\
r_{i}^{\prime}
\end{array}\right]=\left[\begin{array}{l}
e^{\prime \prime} \\
e_{1}^{\prime \prime} \\
\vdots \\
e^{\prime \prime}{ }_{i}
\end{array}\right]
$$

Vector de stock después de la intervención

Después de la intervención (momento $\mathrm{m}_{0}$ ), el vector de stock sufre una reducción a causa del corte y la mortalidad por daño.

Esta reducción puede ser representada matemáticamente de la siguiente manera:

$$
\left[\begin{array}{c}
e_{1}^{\prime} \\
e_{2}^{\prime} \\
\vdots \\
e_{i}^{\prime}
\end{array}\right]=\left[\begin{array}{c}
e_{1}-c_{1}-m_{1} \\
e_{2}-c_{2}-m_{2} \\
\vdots \\
e_{i}-c_{i}-m_{i}
\end{array}\right]
$$

Donde:

$\mathrm{e}_{\mathrm{j}}{ }_{\mathrm{j}}=$ Stock en la clase $\left({ }_{\mathrm{j}}\right)$ en el momento $\left(\mathrm{m}_{0}{ }^{\prime}\right)$ después de la intervención.

$\mathrm{e}_{\mathrm{j}}=$ Stock en la clase $(\mathrm{j})$ en el momento $\left(\mathrm{m}_{0}{ }^{\prime}\right)$.

$\mathrm{c}_{\mathrm{j}}=$ Stock extraído en la clase $(\mathrm{j})$ en el momento $\left(\mathrm{m}_{0}{ }^{\prime}\right)$.

$\mathrm{m}_{\mathrm{j}}=$ mortalidad por daño en la clase $(\mathrm{j})$.

\section{Mortalidad por daño}

Como el objetivo del modelo propuesto es simular el comportamiento del bosque, la mortalidad por daño puede ser estimada por la siguiente fórmula:

Donde:

$$
m_{j}=e_{j} \cdot d \cdot l_{j}
$$

$\mathrm{d}=$ Porcentaje de mortalidad por daño esperada para la primera clase diamétrica, en décimos.

$l_{j}=e_{j} / e_{1}=$ Factor de reducción de la mortalidad en función de la clase de diámetro.

\section{Aumento del reclutamiento}

Con la apertura del dosel habrá un aumento en la regeneración natural y por lo tanto, en el reclutamiento de individuos. La siguiente fórmula matemática tiene como objetivo representar el incremento en el reclutamiento.

Donde:

$$
\left[\begin{array}{c}
r_{1}^{\prime} \\
r_{2}^{\prime} \\
\vdots \\
r_{i}^{\prime}
\end{array}\right]=\left[\begin{array}{c}
r_{1} \cdot h_{i} \\
r_{2} \cdot h_{i} \\
\vdots \\
r_{i} \cdot h_{i}
\end{array}\right]
$$
$\left(\mathrm{m}_{1}\right)$.

$\mathrm{r}_{\mathrm{j}}{ }_{\mathrm{j}}=$ Reclutamiento verificado en la clase $\left({ }_{\mathrm{j}}\right)$ entre el momento $\left(\mathrm{m}_{0}{ }^{\prime}\right)$ después de la intervención y

$r_{j}=$ Reclutamiento verificado entre $\left(m_{0}{ }^{\prime}\right)$ y $\left(m_{1}\right)$.

$\mathrm{h}_{\mathrm{j}}=$ Tasa de aceleración a causa de la apertura del dosel.

La siguiente fórmula determina la tasa de aceleración por clase diamétrica:

$$
\mathrm{h}_{\mathrm{j}}=\left(1+\mathrm{z} \cdot \mathrm{l}_{\mathrm{j}} \cdot \mathrm{w}\right)
$$


Donde:

$\mathrm{z}=$ Tasa de aceleración sobre el reclutamiento de la clase diamétrica 1, en razón de la apertura del dosel.

$\mathrm{l}_{\mathrm{j}}=\mathrm{e}_{\mathrm{j}} / \mathrm{e}_{1}=$ Factor de reducción de la aceleración en función de la clase de diámetro.

$\mathrm{W}=$ Reductor de la tasa de aceleración sobre la tasa $(\mathrm{z})$ en virtud de la recuperación del área basal entre el momento $\left(\mathrm{m}_{\mathrm{t}}\right)$ y $\left(\mathrm{m}_{\mathrm{t}+\mathrm{k}}\right)$, donde $(\mathrm{k})$ representa el intervalo de tiempo de la predicción y el momento actual. El reductor $(\mathrm{w})$ es calculado a través de la siguiente ecuación:

$$
w=\frac{\left(b a_{n}-b a_{m k}\right)}{b a_{c}}
$$

Donde:

$\mathrm{ba}_{\mathrm{n}}=$ Área basal antes de la intervención.

$\mathrm{ba}_{\mathrm{mk}}=$ Área basal después de la intervención en el momento $(\mathrm{k})$.

$\mathrm{ba}_{\mathrm{c}}=$ Área basal retirada siendo $\left(\mathrm{ba}_{\mathrm{mk}}\right)$ siempre menor o igual a $\left(\mathrm{ba}_{\mathrm{n}}\right)$.

\section{Cambio en la matriz de transición}

Según la hipótesis anteriormente descripta, la intervención realizada en el bosque irá a promover un incremento en la tasa de crecimiento diamétrico de los árboles. Por consiguiente la matriz de transición cambia. Habrá un incremento en las probabilidades de transición de una clase a la otra, o sea que la matriz de transición será acelerada.

La nueva matriz (A’) de transición quedará representada de la siguiente manera:

$$
\left[\begin{array}{cccc}
a_{11}^{\prime} & a_{12}^{\prime} & \cdots & a_{1 j}^{\prime} \\
a_{21}^{\prime} & a_{22}^{\prime} & \cdots & a_{2 j}^{\prime} \\
\vdots & \vdots & \cdots & \vdots \\
a^{\prime}{ }_{i 1} & a^{\prime}{ }_{i 2} & \cdots & a_{i j}^{\prime}
\end{array}\right]
$$

Los nuevos valores de los coeficientes serán calculados mediante las siguientes ecuaciones:

- Coeficientes de la diagonal inferior para un período de $\mathrm{m}_{0}-\mathrm{m}_{\mathrm{k}}$.

$$
\mathrm{a}_{\mathrm{i} .}=\mathrm{a}_{\mathrm{i}} \cdot\left(1+\mathrm{t} \cdot \mathrm{l}_{\mathrm{i}} \cdot \mathrm{w}\right)
$$

- Coeficientes de la diagonal principal para un período de $\mathrm{m}_{0}-\mathrm{m}_{\mathrm{k}}$.

Donde:

$$
\mathrm{a}_{\mathrm{i} .}^{\prime}=1-\Sigma \mathrm{a}_{\mathrm{i}}{ }-\mathrm{m}_{\mathrm{i}}
$$

$\mathrm{t}=$ Tasa de aceleración sobre el crecimiento debido a la apertura del dosel.

$\mathrm{l}_{\mathrm{i} . \mathrm{j}}=\mathrm{e}_{\mathrm{j}}+1 / \mathrm{e}_{\mathrm{i}}=$ Factor de reducción de la aceleración en función de la clase de diámetro, siendo $\mathrm{i}>\mathrm{j}$.

$\mathrm{W}=$ Reductor de la tasa de aceleración sobre la tasa $(\mathrm{g})$ debido a la recuperación del área basal en el momento $\left(\mathrm{m}_{\mathrm{k}}\right)$ y $\left(\mathrm{m}_{\mathrm{k}-1}\right)$ donde $(\mathrm{K})$ representa el intervalo entre previsión. Este factor es calculado por la misma fórmula anteriormente descripta.

$\mathrm{m}_{\mathrm{i}}=$ mortalidad normal verificada en la clase (i).

\section{Construcción de las matrices}

Con los datos obtenidos en los años 1998 y 2001 fue determinado, para cada tratamiento y clase diamétrica, el número de árboles que permanecieron, avanzaron a la clase siguiente o murieron, durante el período considerado. Con estos valores fueron calculadas las probabilidades de permanencia en la clase, transición entre clases y mortalidad de los árboles de cada tratamiento y clase diamétrica, necesarias para la construcción de las matrices de transición, el período de proyección fue de tres años. Se obtuvieron matrices para los diferentes tratamientos de cosecha y para las parcelas sin intervención. 
Los resultados de las frecuencias diamétricas, los reclutamientos y los coeficientes para cada una de las matrices, fueron obtenidos a partir de datos promedios sobre el total de parcelas correspondientes a los diferentes tratamientos, logrando de esta manera valores por hectárea. En cuanto a la mortalidad ésta fué incluida en la matriz de transición en la última columna expresando su valor por hectárea.

\section{Predicciones realizadas a través del modelo}

En base a los datos obtenidos en las mediciones correspondientes al período 1998 - 2001 fueron realizadas las predicciones de las distribuciones diamétricas por clase para el período 2001- 2004. Las mismas fueron comparadas con las frecuencias observadas para el mismo año. Se utilizó un test de bondad de ajuste Chi-Cuadrado, a través del cual se compararon si las frecuencias observadas eran estadísticamente homogéneas a las frecuencias estimadas.

\section{RESULTADOS Y DISCUSIÓN}

\section{Obtención de las matrices de transición}

En las tablas 3, 4 y 5 se detallan las matrices de probabilidades para los tratamientos, con sus correspondientes coeficientes y la mortalidad para cada una de las clases diamétricas, para un período de transición de tres años.

Tabla 3. Matriz de Probabilidades de transición después del corte para el tratamiento Cosecha Convencional, por clases diamétricas $(\mathrm{cm})$, para el período 1998 - 2001. Valores por hectárea.

Table 3. Transition matrix probabilities after conventional harvesting, by diameter classes $(\mathrm{cm})$, for the period 1998 - 2001. Values per hectare.

\begin{tabular}{lcccccccc}
\hline \multirow{2}{*}{ Centro de clase diamétrica (cm) } & \multicolumn{7}{c}{ Centro de clase diamétrica (cm) } & \multirow{2}{*}{ Mortalidad } \\
\cline { 2 - 8 } & $\mathbf{1 5}$ & $\mathbf{2 5}$ & $\mathbf{3 5}$ & $\mathbf{4 5}$ & $\mathbf{5 5}$ & $\mathbf{6 5}$ & $\geq \mathbf{7 0}$ & \\
\hline 15 & 0,770 & 0,148 & & & & & & 0,082 \\
25 & & 0,789 & 0,158 & & & & & 0,053 \\
35 & & & 0,809 & 0,131 & & & & 0,060 \\
45 & & & & 0,929 & 0,049 & & & 0,023 \\
55 & & & & & 0,860 & 0,090 & & 0,050 \\
65 & & & & & & 0,755 & 0,195 & 0,050 \\
$\geq 70$ & & & & & & & 0,941 & 0,059 \\
\hline
\end{tabular}

Tabla 4. Matriz de probabilidades de transición después del corte para el tratamiento de Cosecha de Impacto Reducido, por clases diamétricas $(\mathrm{cm})$, para el período 1998 - 2001. Valores por hectárea.

Table 4. Transition matrix probabilities after reduce impact logging, by diameter classes $(\mathrm{cm})$, for the period 1998 - 2001. Values per hectare.

\begin{tabular}{lcccccccc}
\hline \multirow{2}{*}{ Centro de clase diamétrica $(\mathbf{c m})$} & \multicolumn{7}{c}{ Centro de clase diamétrica (cm) } & \multirow{2}{*}{ Mortalidad } \\
\cline { 2 - 8 } & $\mathbf{1 5}$ & $\mathbf{2 5}$ & $\mathbf{3 5}$ & $\mathbf{4 5}$ & $\mathbf{5 5}$ & $\mathbf{6 5}$ & $\geq \mathbf{7 0}$ & \\
\hline 15 & 0,722 & 0,173 & & & & & & 0,104 \\
25 & & 0,799 & 0,102 & & & & & 0,072 \\
35 & & & 0,853 & 0,124 & & & & 0,099 \\
45 & & & & 0,929 & 0,049 & & & 0,023 \\
55 & & & & & 0,805 & 0,110 & & 0,085 \\
65 & & & & & & 0,862 & 0,087 & 0,051 \\
$\geq 70$ & & & & & & & 0,903 & 0,097 \\
\hline
\end{tabular}

Se observó que en todos los tratamientos existe una transición de individuos evolutiva inter e intraclases diamétricas no detectándose estados absorbentes en las mismas.

La probabilidad de muerte de los individuos considerados en este período ocurrió en todas las clases diamétricas en los distintos tratamientos, a excepción de las dos últimas clases diamétricas de las parcelas testigo, donde no se registró esta probabilidad. Puede observarse que las clases diamétricas 
superiores tienen menor número de individuos, característico de la distribución diamétrica de los bosques nativos, entonces al ocurrir una mortalidad en las mismas esta asume un alto valor de probabilidad, respecto de las menores clases diamétricas. En el tratamiento de cosecha de impacto reducido se registraron 7,4 individuos/ha muertos en forma natural siendo éste mayor al registrado en el tratamiento de cosecha convencional (6,2 individuos/ ha).

Tabla 5. Matriz de probabilidades de transición original para las parcelas testigo, por clases diamétricas (cm), para el período 1998 - 2001. Valores por hectárea.

Table 5. Transition matrix probabilities for control plots $(\mathrm{CP})$, by diameter classes $(\mathrm{cm})$, for the period 1998 - 2001. Values per hectare.

\begin{tabular}{lcccccccc}
\hline \multirow{2}{*}{ Centro de clase diamétrica (cm) Centro de clase diamétrica (cm) } & \multirow{2}{*}{ Mortalidad } \\
\cline { 2 - 8 } & $\mathbf{1 5}$ & $\mathbf{2 5}$ & $\mathbf{3 5}$ & $\mathbf{4 5}$ & $\mathbf{5 5}$ & $\mathbf{6 5}$ & $\geq \mathbf{7 0}$ & \\
\hline 15 & 0,871 & 0,082 & & & & & & 0,047 \\
25 & & 0,846 & 0,115 & & & & & 0,038 \\
35 & & & 0,835 & 0,038 & & & & 0,127 \\
45 & & & & 0,882 & 0,059 & & & 0,059 \\
55 & & & & & 0,859 & 0,047 & & 0,094 \\
65 & & & & & & 0,945 & 0,055 & 0,000 \\
$\geq 70$ & & & & & & & 1,000 & 0,000 \\
\hline
\end{tabular}

Respecto a los resultados de los coeficientes obtenidos es necesario una evaluación continua de las probabilidades a lo largo del turno de corta, ya que se cuestiona que las mismas calculadas e insertadas en la matriz permanezcan constantes durante el desarrollo futuro del bosque. Cunha et al., (2002) expresan que es difícil establecer que no habrá cambios en las probabilidades de transición en el tiempo, especialmente en casos de poblaciones de árboles que viven períodos largos.

\section{Ingresos por clases diamétricas para cada tratamiento}

En el siguiente cuadro se detallan los ingresos pertenecientes al período 1998 - 2001 para cada tratamiento (Tabla 6).

Tabla 6. Vector de ingreso para los tratamientos de cosecha y para las parcelas testigos.

Table 6. Recruitment for the harvesting treatments and for control plots.

\begin{tabular}{lccc}
\hline $\begin{array}{l}\text { Centro de clases } \\
\text { diamétricas }(\mathbf{c m})\end{array}$ & \multicolumn{3}{c}{ Número de ingresos (Individuos/ha) } \\
\cline { 2 - 4 } & Cosecha convencional & Cosecha de impacto reducido & Testigo \\
\hline 15 & 5 & 7 & 12 \\
25 & 1 & 2 & 5 \\
35 & 0 & 0 & 0 \\
45 & 0 & 0 & 0 \\
55 & 0 & 0 & 0 \\
65 & 0 & 0 & 0 \\
$\geq 70$ & 0 & 0 & 0 \\
\hline Total & 6 & 9 & 17 \\
\hline
\end{tabular}

Respecto a los tratamientos de cosecha no se detectó un aumento inmediato en el vector ingreso respecto a la testigo. En las parcelas testigos se registró un mayor número de ingresos por hectárea. Esto hace suponer que el período considerado de tres años no fue suficiente para detectarlos. Ello es coincidente con un análisis realizado sobre la base de datos del proyecto ( 7 años) donde se visualiza que este aumento se comienza a magnificar entre el quinto y sexto año de realizada la explotación.

\section{Análisis de las predicciones para cada tratamiento}

A continuación se presentan los valores de Chi-cuadrado teóricos y calculados para cada tratamiento (Tabla 7). 
Tabla 7. Valores de Chi-Cuadrado teóricos y calculados, X.95.

Table 7. Chi- squared test for theoretical and calculated values, X.95.

\begin{tabular}{lccc}
\hline Tratamiento & $\begin{array}{c}\text { Grados de } \\
\text { Libertad }\end{array}$ & $\begin{array}{c}\text { Valores Chi-Cuadrado } \\
\text { teóricos }\end{array}$ & $\begin{array}{c}\text { Valores Chi-Cuadrado } \\
\text { calculados }\end{array}$ \\
\hline Cosecha convencional & 5 & 11,07 & 0,44 \\
Cosecha de impacto reducido & 5 & 11,07 & 0,08 \\
Testigo & 6 & 12,59 & 1,37 \\
\hline
\end{tabular}

El test de bondad de ajuste Chi-Cuadrado, muestra que a un nivel de 5\% de probabilidad para cada uno de los tratamientos las predicciones de las frecuencias diamétricas por clase correspondientes al período 2001- 2004 son estadísticamente homogéneas con las frecuencias observadas para el mismo año (2004).

Las figuras 1a, $1 \mathrm{~b}$ y $1 \mathrm{c}$ representan las frecuencias observadas y predichas correspondiente al año 2004, para los diferentes tratamientos de cosecha y para las parcelas testigos.

Se observa en la figura 1 que tanto en los tratamientos de cosecha como en las parcelas testigos, mantienen la forma básica de la distribución de "J" invertida, característico de los montes nativos.

\section{Resultados de las proyecciones y evolución de los parámetros para el manejo del monte nativo}

En las tablas 8, 9 y 10 se presentan las proyecciones obtenidas para cada tratamiento de cosecha y para las parcelas testigos respecto a la evolución del área basal $\left(\mathrm{AB} \mathrm{m}^{2} / \mathrm{ha}\right)$ y el número de individuos por hectárea $(\mathrm{N})$.

Tabla 8. Evolución de los parámetros para el tratamiento de cosecha convencional, por hectárea.

Table 8. Parameters evolution, treatment conventional harvesting, values per hectare.

\begin{tabular}{|c|c|c|c|c|c|c|c|c|c|c|c|}
\hline Parámetros & 1998 & 1999 & 1998' & 2001 & 2004 & 2007 & 2010 & 2013 & 2016 & 2019 & 2022 \\
\hline $\begin{array}{l}\text { AB antes de la } \\
\text { intervención }\left(\mathrm{m}^{2} / \mathrm{ha}\right)\end{array}$ & 13,37 & & & & & & & & & & \\
\hline $\begin{array}{l}\text { AB después de la } \\
\text { intervención }\left(\mathrm{m}^{2} / \mathrm{ha}\right)\end{array}$ & & & 10,61 & 11,01 & 11,39 & 11,75 & 12,1 & 12,43 & 12,75 & 13,05 & 13,34 \\
\hline $\mathrm{AB}$ extraída $\left(\mathrm{m}^{2} / \mathrm{ha}\right)$ & & 1.90 & & & & & & & & & \\
\hline $\begin{array}{l}\text { AB muerta por daño } \\
\left(\mathrm{m}^{2} / \mathrm{ha}\right)\end{array}$ & & 0.86 & & & & & & & & & \\
\hline $\begin{array}{l}\text { Reductor de la tasa } \\
\text { de aceleración (W) }\end{array}$ & & & 1,00 & 0,86 & 0,72 & 0,59 & 0,46 & 0,34 & 0,22 & 0,12 & 0,01 \\
\hline $\begin{array}{l}\text { Número individuos } \\
\text { por hectárea }(\mathrm{N})\end{array}$ & 100,82 & 7,93 & 92,89 & 93,96 & 95,03 & 96,08 & 97,1 & 98,14 & 99,14 & 100,1 & 101,1 \\
\hline
\end{tabular}

Tabla 9. Evolución de los parámetros para el tratamiento de cosecha de impacto reducido, por hectárea.

Table 9. Parameters evolution, treatment reduced impact logging, values per hectare.

\begin{tabular}{|c|c|c|c|c|c|c|c|c|c|c|}
\hline Parámetros & 1998 & 1999 & $1998 '$ & 2001 & 2004 & 2007 & 2010 & 2013 & 2016 & 2019 \\
\hline $\begin{array}{l}\text { AB antes de la } \\
\text { intervención }\left(\mathrm{m}^{2} / \mathrm{ha}\right)\end{array}$ & 12,19 & & & & & & & & & \\
\hline $\begin{array}{l}\text { AB después de la } \\
\text { intervención }\left(\mathrm{m}^{2} / \mathrm{ha}\right)\end{array}$ & & & 10,13 & 10,48 & 10,77 & 11,05 & 11,3 & 11,59 & 11,84 & 12,09 \\
\hline AB extraída (m²/ha) & & 1.36 & & & & & & & & \\
\hline $\begin{array}{l}\text { AB muerta por daño } \\
\left(\mathrm{m}^{2} / \mathrm{ha}\right)\end{array}$ & & 0.7 & & & & & & & & \\
\hline $\begin{array}{l}\text { Reductor de la tasa } \\
\text { de aceleración (W) }\end{array}$ & & & 1,00 & 0,83 & 0,69 & 0,55 & 0,42 & 0,29 & 0,17 & 0,05 \\
\hline $\begin{array}{l}\text { Número de } \\
\text { individuos por } \\
\text { hectárea }(\mathrm{N})\end{array}$ & 95,10 & 5,64 & 89,46 & 91,67 & 93,04 & 94,4 & 95,6 & 96,72 & 97,72 & 98,63 \\
\hline
\end{tabular}


Tabla 10. Evolución de los parámetros para las parcelas sin intervención por hectárea.

Table 10. Parameters evolution, control plots, values per hectare.

\begin{tabular}{lccccccccc}
\hline Parámetros & $\mathbf{1 9 9 8}$ & $\mathbf{2 0 0 1}$ & $\mathbf{2 0 0 4}$ & $\mathbf{2 0 0 7}$ & $\mathbf{2 0 1 0}$ & $\mathbf{2 0 1 3}$ & $\mathbf{2 0 1 6}$ & $\mathbf{2 0 1 9}$ & $\mathbf{2 0 2 2}$ \\
\hline $\begin{array}{l}\text { Área basal inicial } \\
\left(\mathrm{m}^{2} / \text { ha }\right)\end{array}$ & 15,04 & 15,01 & 15,20 & 15,47 & 15,78 & 16,12 & 16,48 & 16,85 & 17,24 \\
$\begin{array}{l}\text { Número de individuos } \\
\text { por hectárea (N) }\end{array}$ & 119,60 & 120,97 & 127,56 & 132,63 & 143,41 & 150,89 & 158,05 & 164,87 & 171,36 \\
\hline
\end{tabular}

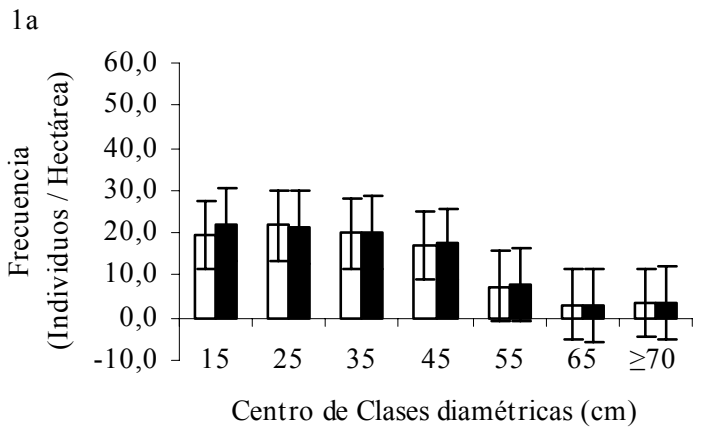

$\square$ Frecuencias Observadas $\square$ Frecuencias Predichas

$1 \mathrm{~b}$

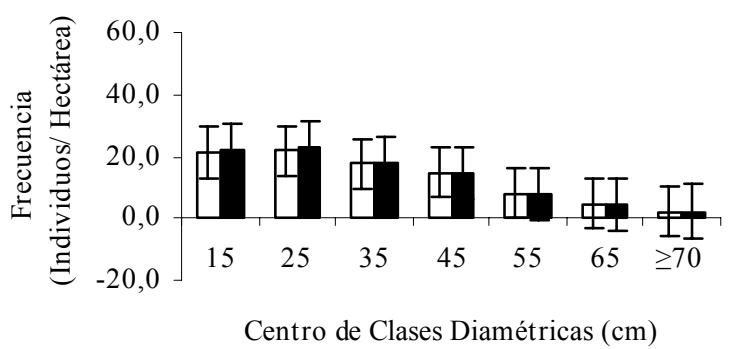

$\square$ Frecuencias Observadas $\square$ Frecuencias Predichas

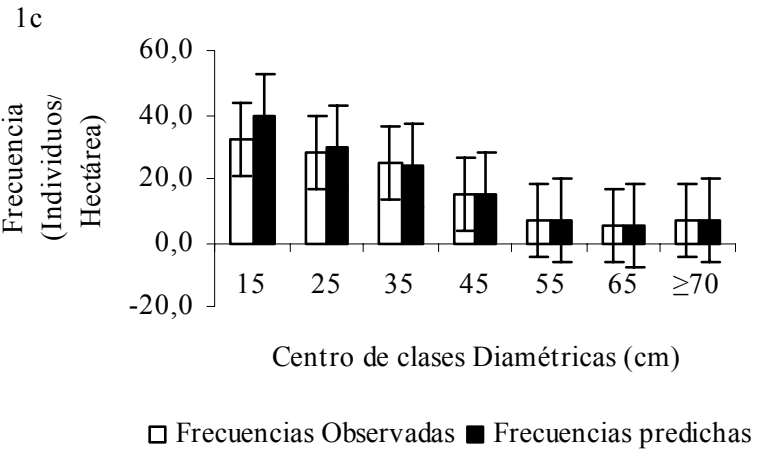

Figura 1. Frecuencias observadas y predichas: a: tratamiento de cosecha convencional; b: tratamiento de cosecha de impacto reducido; c: parcelas testigos.

Figure 1. Observed and estimated frequencies: a: conventional harvesting; b: reduced impact logging; c: control plots. 
Según Mac Donagh et al. (2003), para el mismo ensayo, encontraron que no existen diferencias significativas tanto en el área basal $\left(1,90 \mathrm{~m}^{2}\right.$ ha- 1 en cosecha convencional; $1,36 \mathrm{~m}^{2}$ ha- 1 en cosecha de impacto reducido; $p=0,30)$ como en el número de árboles extraídos (4,3 ha-1 en cosecha convencional; 2,8 ha- 1 en cosecha de impacto reducido; $p=0,17$ ) en los tratamientos de cosecha.

Puede observarse que el área basal original es recuperada a los 24 años para el tratamiento de cosecha convencional y a los 21 años para el tratamiento de impacto reducido; esta diferencia en los mismos puede ser explicada por la mayor mortalidad por daños que hubo en el tratamiento de cosecha convencional, motivo por el cual se retrasa la recuperación del área basal. En ambos tratamientos de cosecha se observa que los individuos muertos por la explotación fueron mayores en las primeras clases diamétricas tornándose nulos en las últimas. Louman et al. (2001) señalan que aprovechamientos bien planificados y con un buen control de las operaciones, se traducen en menores costos de operación y menores daños al bosque remanente. Se puede observar que el tratamiento de cosecha de impacto reducido a través de una planificación correcta de cosecha disminuye los daños principalmente a las especies de interés comercial asegurando de esta forma la permanencia para futuras cosechas.

Teniendo en cuenta el número de individuos se puede observar que el tratamiento de cosecha de impacto reducido se recupera a los 12 años de realizado el aprovechamiento, en cambio el de cosecha convencional lo hace a los 21 años, esto puede deberse a que el registro de los ingresos en el de cosecha de impacto reducido fue mayor.

En cuanto al reductor de la tasa de aceleración (W) puede observarse que se modifica a medida que el monte nativo recupera su área basal original en ambos tratamientos.

Analizando la evolución de los parámetros en los tratamientos de cosecha con las áreas testigos se puede inferir que en los tratamientos de cosecha hubo una recuperación diferencial en el área basal y número de individuos en comparación con los valores originales. Sin embargo, el bosque sin intervención siguió creciendo, con lo cual aquellos valores de recuperación no se acercan a los de la testigo cuando es considerado un plazo de veinte años. Otro aspecto a tener en cuenta serían las estimaciones de los datos de ingreso y mortalidad. Sanquetta et al. (1995) expresan que son necesarios desarrollar ecuaciones correlacionando la mortalidad y los ingresos con características de la población, para asegurar esta confiabilidad en las estimaciones de estos eventos.

\section{CONCLUSIONES}

Las matrices de transición constituyen una herramienta valiosa para el manejo del bosque. A través de las mismas se pudo analizar la estructura diamétrica y demostrar una evolución de la masa remanente después de la explotación en función de los tratamientos.

Las matrices produjeron estimaciones satisfactorias de las distribuciones diamétricas para un período de tres años para los distintos tratamientos de cosecha, como así también para las parcelas testigos.

A través de las simulaciones efectuadas queda demostrado que el tratamiento de cosecha convencional recupera su área basal original a los 24 años de realizado el aprovechamiento y el número de individuos a los 21 años. En cambio el de cosecha de impacto reducido recupera su área basal original a los 21 años y el número de individuos a los 12 años de realizado el aprovechamiento. La disminución de los daños del tratamiento de cosecha de impacto reducido se reflejó en un acortamiento de los turnos de corta y en la recuperación del bosque de forma más sustentable que el de cosecha convencional. En cuanto a las parcelas testigos se registró un crecimiento en la estructura arbórea; las áreas intervenidas alcanzaron los valores originales pero no los del bosque sin intervención, esto cuando considerado un plazo de veinte años.

La construcción de matrices de transición es una metodología sencilla, fácil de aplicar y tiene la ventaja de que pueden ser construidas con tan solo dos mediciones.

\section{AGRADECIMIENTOS}

Los autores agradecen al CIFOR (Centro Internacional para la Investigación Forestal) y al Japan Research Project por el apoyo financiero. Al Ingeniero Forestal Julio Arce por las sugerencias en el desarrollo del presente trabajo. 


\section{REFERENCIAS}

AZEVEDO, C. P.; SOUZA, A. L.; CAMPOS, J. C.; PAULA JUNIOR, G. Predição da distribuição diamétrica da floresta Atlântica pelo emprego da matriz de transição. Árvore, Viçosa, v. 18, n. 3, p. 179193, 1994.

CUNHA, U. S.; MACHADO, S. A.; FIGUEIREDO FILHO, A.; SANQUETTA, C. R. Predição da estrutura diamétrica de espécies comerciais de Terra Firme da Amazônia por meio da matriz de transição. Ciência Florestal, Santa Maria, v. 12, n. 1, p. 109-122, 2002.

DYKSTRA, D. P.; HEINRICH, R. Model Code of Forest Harvesting Practice. Food and Agriculture Organization of the United Nations (FAO), Rome, 1996, 85 p.

GARIBALDI, J.; RIVERO, L.; MAC DONAGH, P. Structure, floristic composition and spatial distribution of tree species in an unlogged forest of the Alto Paraná Atlantic Forest In: MAC DONAGH, P. Forest harvesting impacts evaluation on forest ecosystems final report for phase I. FCF. UNaM. CIFOR. 2000, 70 p.

LOUMAN, B.; QUIRÓS, D.; NILSON, M. Silvicultura de bosques latifoliados húmedos con énfasis en América Central. CATIE. Manual Técnico n. 46, p. 134-136, 2001.

MAC DONAGH, P.; GARIBALDI, J.; RIVERO, L.; SNOOK, L.; TOMA, T. Comparación de daños, mortalidad y reclutamiento en dos sistemas de aprovechamiento forestal en Misiones, Argentina. In: $\mathbf{X}$ Jornadas Téenicas Forestales y Ambientales. Facultad de Ciencias Forestales - Universidad Nacional de Misiones. Instituto Nacional de Tecnología Agropecuaria. Eldorado Misiones. En CD. 2003

MAC DONAGH, P.; GAUTO, O.; LÓPEZ CRISTÓBAL, L.; VERA, N.; FIGUEREDO, S.; FERNÁNDEZ, R.; GARIBALDI, J.; ALVEZ, M.; KELLER, H.; MAREK, M.; CAVALLIN, J.; KOBAYASHI, S. 2001. Evaluation of forest harvesting impacts on forest ecosystems. In: KOBAYASHI, S.; TURNBULL W.; TOMA, T.; MORI, T.; MAJID NMNA (eds) Rehabilitation of degrades tropical forest ecosystems. Workshop proceedings. Bogor. p. 69-79, 1999.

MORALES, E. H. Diseño experimental a través del análisis de varianza y modelos de regresión lineal. Primera Edición, 2005, 252 p.

PUlZ, F.; SCOLFORO, J.; OlIVEIRA, A.; MELlO, J.; OLIVEIRA FILHO, A. Acuracidade da predição da distribuição diamétrica da uma floresta inequiânea com a matriz de transição. Cerne, Lavras, v. 5, n. 1, p. 01-14, 1999.

SANQUETTA, C. R.; ANGELO, H.; BRENA, D. A.; MENDES, J. Predição da distribuição diamétrica, mortalidade e recrutamento da floresta natural com matriz markoviana de potência. Floresta, Curitiba, v. 24, n. 1/2, p. 23-37, 1995.

SANQUETTA, C. R.; BRENA, D. A.; ANGELO, H. Matriz de transição para simulação da dinâmica de florestas naturais sob diferentes intensidades de corte. Ciência Florestal, Santa Maria, v. 6, n. 1, p. 65-78, 1996.

SCOLFORO, J. Modelagem da producão, idade das florestas nativas, distribuicão espacial das espécies e análise estructural. Manejo Forestal. UFLA / FAEPE: Lavras. p. 210 - 217, 1997. 